\title{
Physicochemical characteristics and antioxidant activity of the intracellular melanin from Lachnum sp. YM-236
}

\author{
Gengyi Guo ${ }^{a}$, Ming Ye ${ }^{b *}$, Meishuang Qianc ${ }^{c}$ Qingping Xu ${ }^{d}$, Liu Yang ${ }^{\mathrm{e}}$ \\ College of Biotechnology and Food Engineering, Hefei University of Technology, Heifei 230009, \\ Anhui, China \\ aguogengyi@126.com; byeming123@sina.com(*corresponding author); 'qianmeishuang@163.com; \\ dxuqingping@163.com; eyangliu199@163.com.
}

Keywords: Lachnum; eumelanin; physicochemical characteristics; antioxidant activity

\begin{abstract}
Melanin LEM236 was extracted and purified from Lachnum YM-236. Scanning electron microscopy showed LEM236 had a blocky, amorphous crystal structure with an irregular surface. Elemental analysis, infrared spectroscopy and ${ }^{1} \mathrm{H}-\mathrm{NMR}$ measurements indicated that the melanin had the structure model of a novel eumelanin-like pigment. The total antioxidant capacity of $1 \mathrm{~g} / \mathrm{L}$ the melanin was equivalent to that of $53.28 \pm 0.18 \mathrm{mmol} / \mathrm{L} \alpha$-tocopherol. $\mathrm{IC}_{50}$ values of LEM236 for scavenging activity of $\mathrm{ABTS}^{+}, \cdot \mathrm{OH}$ and $\mathrm{NO}_{2}^{-}$were $0.680 \mathrm{~g} / \mathrm{L}, 0.759 \mathrm{~g} / \mathrm{L}$ and $0.839 \mathrm{~g} / \mathrm{L}$, respectively.
\end{abstract}

\section{Introduction}

Melanin is a polyphenolic polymer which has such functions as oxidation resistance, free radical scavenging and anti-aging [1]. To produce melanin via microbial fermentation has the advantages of being easily controlled, needing mild reaction conditions and not being impacted by geographical and climatic conditions. Lachnum is a category of saprophytic fungi that distributed throughout the world, and had been discovered could produce a great amount of melanin under submerged culture conditions. This melanin is stable to temperature, light, $\mathrm{UV}, \mathrm{Na}_{2} \mathrm{SO}_{3}$ and sodium benzoate, having the structure model of both eumelanin and pheomelanin [2].

The aim of the present work was to prepare LEM236 from Lachnum YM-236, reveal its physicochemical characteristics, and assess its antioxidant activity in vitro.

\section{Materials and methods}

\section{Materials}

Sporocarps of Lachnum YM-236 was separated and preserved by the Laboratory of Microbial Resources and Application of Hefei University of Technology.

\section{Extraction and purification of LEM236}

The method of Wang et al [3] with minor improvements was used. The dried mycelium was mixed with $0.5 \mathrm{~mol} / \mathrm{L} \mathrm{NH}_{3} \cdot \mathrm{H}_{2} \mathrm{O}$ at the proportion of $1: 40(\mathrm{w} / \mathrm{v})$, extracted in water bath at $80{ }^{\circ} \mathrm{C}$ for $5 \mathrm{~h}$, The water extracts were centrifuged and the supernatants were pooled and acidified with $6 \mathrm{M}$ $\mathrm{HCl}$ to $\mathrm{pH} 2.0$. The precipitate was successively extracted with chloroform, ethyl acetate and absolute ethanol after hydrolyzed by $6 \mathrm{~mol} / \mathrm{L} \mathrm{HCl}$ solution at $100{ }^{\circ} \mathrm{C}$ for $4 \mathrm{~h}$. The purified melanin was obtained after vacuum freeze-drying.

\section{Physicochemical characteristics of LEM236}

The method of Ye et al [4] was used for scanning electron microscopy, infrared spectroscopy, ${ }^{1}$ H-NMR spectroscopy and elemental analysis of LEM236. The morphological features of the sample were imaged by a KYKY2800 scanning electron microscope (SEM) (KYKY Technology Development Ltd, China) at working voltage $20 \mathrm{kV} / 25 \mathrm{kV}$ and working distance $6.0 \mathrm{~mm}$. The percentages of $\mathrm{C}, \mathrm{H}$ and $\mathrm{N}$ in the intracellular melanin were determined using an elemental analyzer (Elementar Vario EL elemental analyzer, Elementaranalysen systeme, Hanau, Germany). 5700 FT-IR spectrometer (ThermoElectron, Madison, WI, USA) was used for infrared spectroscopy 
between $4000-400 \mathrm{~cm}^{-1}$. And the ${ }^{1} \mathrm{H}-\mathrm{NMR}$ spectra of the sample was recorded at physiological temperature of $298 \mathrm{~K}$ on a Bruker Avance AV-400 spectrometer operating at $500.13 \mathrm{MHz}$.

\section{Antioxidant activity of LEM236}

Total antioxidant capacity (TAC) assay of LEM236. Measurement of the total phenolic content was assessed by using the method of Turkoglu et al [5]. The total antioxidant capacity was expressed by the $\alpha$-tocopherol equivalent: $A=0.011 C+0.0049\left(\mathrm{R}^{2}=0.987\right)$, where $\mathrm{A}$ was the absorbance value at $695 \mathrm{~nm}$, and $\mathrm{C}$ was the equivalent concentration of $\alpha$-tocopherol $(\mathrm{mmol} / \mathrm{L})$.

Effect of scavenging ABTS ${ }^{+}$of LEM236. The ability of LEM236 to scavenge ABTS $\cdot^{+}$free radical was determined by the method of Zhang et al [6]. When the reaction was completed, the absorbance was measured at $560 \mathrm{~nm}$. The distilled water was used instead of the sample as control. ABTS $\cdot^{+}$scavenging rate $(\%)=\left(1-\mathrm{A}_{\text {sample }} / \mathrm{A}_{\text {control }}\right) \times 100 \%$.

$\cdot \mathrm{OH}$ scavenging assay of LEM236. $\mathrm{OH}$ scavenging activity was assessed by using the method of Ye et al [7]. The undamaged tubes were not added with $\mathrm{H}_{2} \mathrm{O}_{2}$, melanin solution or Vc solution. $\cdot \mathrm{OH}$ scavenging rate $(\%)=\left[\left(\mathrm{A}_{\text {sampled }}-\mathrm{A}_{\text {damaged }}\right) /\left(\mathrm{A}_{\text {undamaged }}-\mathrm{A}_{\text {damaged }}\right)\right] \times 100$.

$\mathrm{NO}_{2}^{-}$(nitrite) scavenging assay of LEM236. $\mathrm{NO}_{2}^{-}$scavenging assay was determined using a method of Jiao et al [8]. Sodium nitrite scavenging rate $\mathrm{Sa}(\%)=\left[\mathrm{A}_{\mathrm{s}}-\left(\mathrm{A}_{\mathrm{p}}-\mathrm{A}_{\mathrm{c}}\right)\right] / \mathrm{A}_{\mathrm{s}} \times 100$. Where $\mathrm{A}_{\mathrm{c}}$ was the absorbance of the control group, $A_{s}$ was the absorbance of the standard sample.

\section{Results and discussion}

\section{Physicochemical characteristics of LEM236}

As shown in Fig.1, LEM236 had a blocky, amorphous crystal structure with an irregular surface, which is different from the spherical sepia melanin [9]. The percentages of $\mathrm{C}, \mathrm{H}$ and $\mathrm{N}$ in LEM236 were $54.33 \pm 0.24 \%, 4.168 \pm 0.054 \%$ and $5.286 \pm 0.0335 \%$, respectively. Compared with the Dopa melanin (eumelanin) and brown melanin (Table 1) reported by Ito and Fujita, LEM236 contained less $\mathrm{N}$ and had a higher $\mathrm{C}$ : $\mathrm{N}$ ratio, indicating that LEM236 might contain aliphatic groups [10].

Infrared spectroscopy is the spectroscopy frequently used to examine the organic structure. It does not destroy the sample structure and can reveal the detailed information about functional groups of the sample. Fig. 2 gives the infrared spectra of LEM236, which shows the following structural information of the melanin: $\mathrm{N}-\mathrm{H}$ stretching vibration in the indole ring $\left(3412 \mathrm{~cm}^{-1}\right), \mathrm{CH}_{3}$ asymmetric stretching vibration $\left(2932 \mathrm{~cm}^{-1}\right)$, stretching vibrations of $\mathrm{COOH}, \mathrm{C}=\mathrm{O}$ or $\mathrm{C}=\mathrm{C}(1627$ $\left.\mathrm{cm}^{-1}\right)$, C-N stretching vibration $\left(1398 \mathrm{~cm}^{-1}\right)$, COC asymmetric stretching vibration $\left(1128 \mathrm{~cm}^{-1}\right)$. These results indicated that LEM236 contains benzoquinone structure and indole structure.

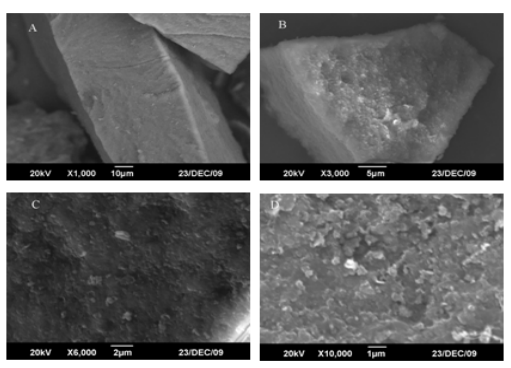

Fig.1 SEM image of LEM236

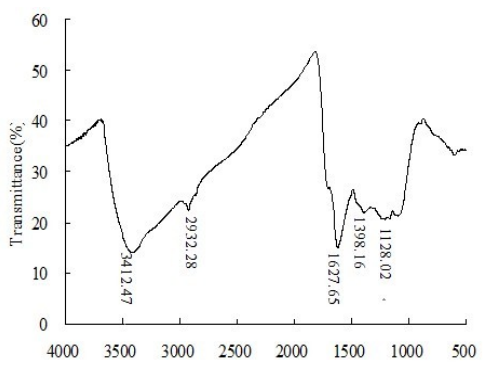

Fig. 2 Infrared spectrum of LEM236

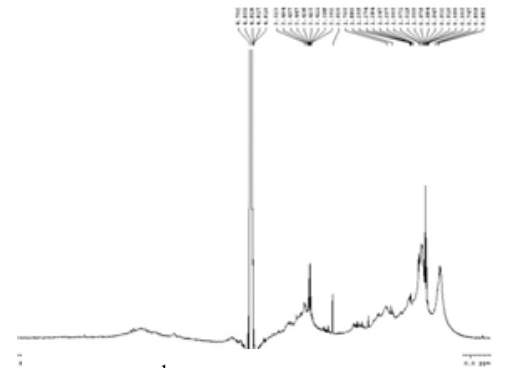

Fig. $3{ }^{1}$ H-NMR spectrum of LEM236

${ }^{1}$ H-NMR spectrum of LEM236 presented a series of broad peaks, which was similar with melanin from Gliocephalotrichum simplex and Lachnum singerianum [1, 4]. Signals in the 2.2-2.5 ppm and 2.5-3.5 ppm region were assigned to $\mathrm{CH}_{3}$ group and $\mathrm{NH}$ group respectively that are connected to the indole groups. The resonance at 3.5-4.0 ppm was assigned to $\mathrm{CH}$ group that is connected to NH group. Absorption peak in the 7.0-7.5 ppm region may correspond to the indole or other aromatic heterocyclic ring of the melanin polymer chain. 
Table 1 Elemental analysis for different melanins

\begin{tabular}{|c|c|c|c|}
\hline & \multicolumn{3}{|c|}{ Content (\%) } \\
\cline { 2 - 4 } & $\mathrm{C}$ & $\mathrm{H}$ & $\mathrm{N}$ \\
\hline LEM236 & 54.33 & 4.168 & 5.286 \\
\hline Dopa melanin (eumelanin)* & 56.45 & 3.15 & 8.49 \\
\hline Brown melanin* & 46.24 & 4.46 & 9.36 \\
\hline
\end{tabular}

*Its data is from Ito and Fujita' report (1985)

\section{Antioxidant activity of LEM236}

The total phenolic content (TPC) and total antioxidant capacity (TAC) of LEM236 both increased with the increase of the LEM 236 concentration, exhibiting a good dose-effect relationship $\left(y=120.52 x-7.8523, R^{2}=0.9812 ; y=43.846 x+10.803, R^{2}=0.9615\right)$ (Fig.4-A and Fig. 4-B). The TAC of $1 \mathrm{~g} / \mathrm{L}$ LEM236 was equivalent to $53.28 \pm 0.18 \mathrm{mmol} / \mathrm{L} \alpha$-tocopherol, and the TAC of $1 \mathrm{~g} / \mathrm{L} \mathrm{BHA}$ was equivalent to $176.3 \mathrm{mmol} / \mathrm{L}$ - -tocopherol. As shown in Fig.4-C, there was a good linear correlation between the phenolic content and the antioxidant activity of LEM236 $\left(y=2.6182 x-32.961, R^{2}=0.9256\right)$, which was consistent with the result of Kumaran et al [11].
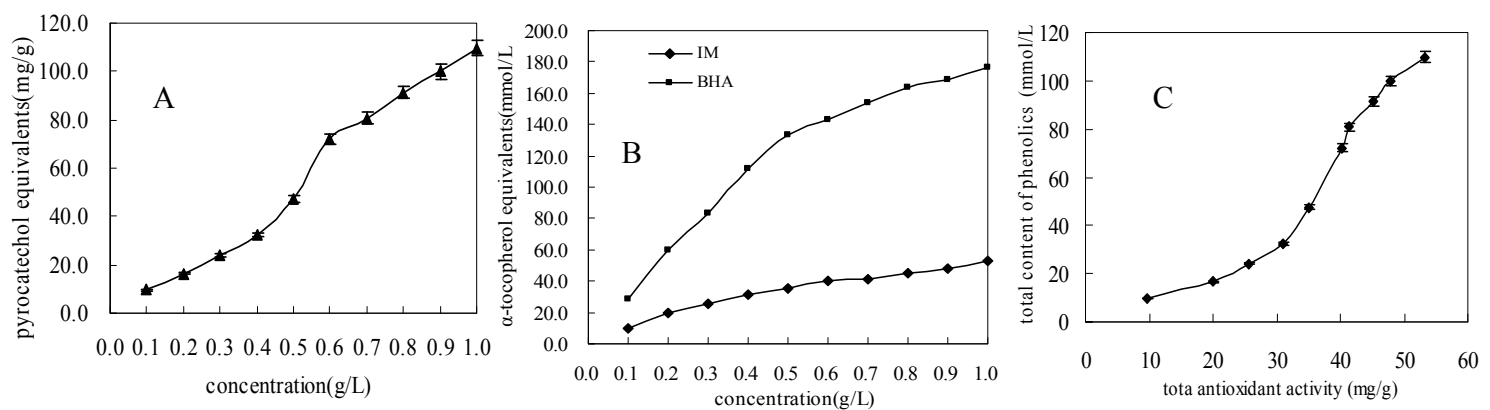

Fig.4 Total phenolic contents (A), total antioxidant capacity (B) and its correlation (C) of LEM236

The scavenging effects of ABTS ${ }^{+}$and $\cdot \mathrm{OH}$ were shown in Fig.5-A and Fig.5-B. Both of them had good linear correlations between the content and the antioxidant activity of LEM236 $\left(\mathrm{y}=80.886 \mathrm{x}-4.9679, \mathrm{R}^{2}=0.9814 ; \mathrm{y}=83.681 \mathrm{x}-13.521, \mathrm{R}^{2}=0.9388\right)$. Compared with the control BHT, LEM236 had a lower ABTS. ${ }^{+}$scavenging activity, with the $\mathrm{IC}_{50}$ value being $0.680 \mathrm{~g} / \mathrm{L}$. And the hydroxyl radical scavenging activity of LEM236 was lower than that of $\mathrm{Vc}$ or BHA. $\mathrm{IC}_{50}$ value of LEM236 was $0.759 \mathrm{~g} / \mathrm{L}$, whereas $\mathrm{IC}_{50}$ values of $\mathrm{Vc}$ and $\mathrm{BHA}$ were $0.677 \mathrm{~g} / \mathrm{L}$ and $0.570 \mathrm{~g} / \mathrm{L}$, respectively. Fig.5-C shows that, with the increase of the LEM236 concentration, the $\mathrm{NO}_{2}{ }^{-}$ scavenging ability of LEM236 gradually increased, having a lower scavenging activity than Vc or BHA of the control groups at the same concentration. $\mathrm{IC}_{50}$ value of $\mathrm{LEM} 236$ for $\mathrm{NO}_{2}{ }^{-}$was $0.839 \mathrm{~g} / \mathrm{L}$, and $\mathrm{IC}_{50}$ values of BHA and $\mathrm{Vc}$ were $0.145 \mathrm{~g} / \mathrm{L}$ and $0.184 \mathrm{~g} / \mathrm{L}$, respectively.
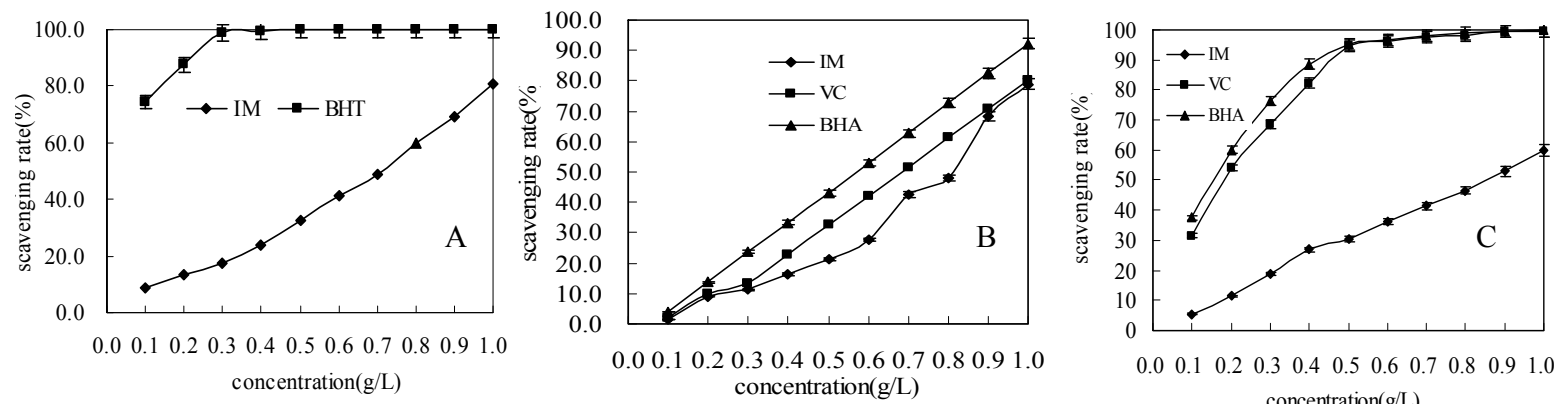

Fig.5 $\mathrm{ABTS}^{+}(\mathrm{A}), \cdot \mathrm{OH}(\mathrm{B})$ and $\mathrm{NO}_{2}^{-}(\mathrm{C})$ scavenging activity of LEM236

\section{Conclusions}

It was concluded that, based on the analysis of elemental analysis, infrared spectroscopy and ${ }^{1}$ H-NMR spectroscopy, LEM236 had the structure model of a novel eumelanin-like pigment. Scanning electron microscopy showed LEM236 had a blocky, amorphous crystal structure with an irregular surface. It had strong scavenging activity against $\mathrm{ABTS} \cdot{ }^{+}, \cdot \mathrm{OH}$ and $\mathrm{NO}_{2}{ }^{-}$, indicating that the melanin had a high antioxidant activity, and can be expected to be used as a new antioxidant in such industries as food and medicine. 


\section{Acknowledgments}

This work was financially supported by the National Natural Science Foundation of China (31070021; 31270000) and Scientific Research Foundation of Year-Plan in Anhui Province (1202030300)

\section{References}

[1] P. Jalmi, P.Bodke, S.Wahidullah, S.Raghukumar: World J Microb Biot. Vol. 28 (2012), p. 505

[2] M.Ye, W.Tan, H.Chen, J.Zhao, C.M.Tang: Food Sci. Vol. 28 (2007), p. 229

[3] H.S.Wang, Y.M.Pan, X.J.Tang, Z.Q.Huang: LWT-Food Sci Technol. Vol. 9 (2006), p. 496

[4] M.Ye, X.Chen, G.W.Li, G.Y.Guo, L.Yang: Adv Mater Res. Vol. 284 (2011a), p. 1742

[5] A.Turkoglu, M.E.Duru, N.Mercan, I.Kivrak, K.Gezer: Food Chem. Vol. 101 (2007), p. 267

[6] Q.F.Zhang, Z.R.Zhang, H.Y.Cheung: Food Chem. Vol. 115 (2009), p. 297

[7] M.Ye, Y.Wang, M.S.Qian, X.Chen, X.Q.Hu: IJBAS-IJENS. Vol. 11 (2011b), p. 51

[8] Z.G.Jiao, J.C.Liu, S.X.Wang: Sci and Technol of Food Ind. Vol. 25 (2004), p. 127

[9] Y.Liu, J.D.Simon: Pigm Cell Res. Vol. 16 (2003), p. 72

[10] S.Ito, K.Fujita: Anal Biochem. Vol. 144 (1985), p. 527

[11] A.Kumaran, J.Karunakaran: LWT-Food Sci Technol. Vol. 40 (2006), p. 344 